\title{
An overall and dose-response meta-analysis for thyrotropin and thyroid cancer risk by histological type
}

\author{
Na Hu${ }^{1,2, *}$, Zhan-Ming Li ${ }^{3, *}$, Jin-Feng Liu ${ }^{1,2}$, Zhen-Zhen Zhang ${ }^{1,2}$, Li-Shun Wang ${ }^{1}$ \\ ${ }^{1}$ Institute of Biomedical Sciences, Minhang Hospital, Fudan University, Shanghai, P.R. China \\ ${ }^{2}$ School of Public Health Taishan Medical University, Shandong, P.R. China \\ ${ }^{3}$ Ruijin Hospital, Shanghai Jiao-Tong University School of Medicine, Shanghai, P.R. China \\ *These authors contributed equally to this work
}

Correspondence to: Li-Shun Wang, email: lishunwang@fudan.edu.cn

Keywords: thyroid cancer, serum thyrotropin, meta-analysis, differentiated thyroid carcinoma, papillary thyroid carcinoma

Received: December 16, 2015

Accepted: June 12, 2016

Published: June 24, 2016

\section{ABSTRACT}

Thyrotropin (TSH) is thought as a risk factor for thyroid cancer. However, the effect of serum TSH might depend on histological types of thyroid cancer. We searched for related studies including serum TSH as an exposure and thyroid cancer as a result in PUBMED, EMBASE and Chinese National Knowledge Infrastructure up to April 21, 2016. This meta-analysis included 22 articles with 53,538 participants. When comparing all histological thyroid cancer, the pooled odds ratios of thyroid cancer in patients with nodules was found to increase significantly with higher serum TSH concentrations for differentiated thyroid carcinoma (1.88 vs $.1 .48, P=0.0000)$ and papillary thyroid carcinoma ( 2.08 vs. $1.48, P=0.0006)$. Each $1 \mathrm{mU} / \mathrm{L}$ increase of serum TSH was associated with $14 \%$ greater risk of thyroid cancer for all histological thyroid cancer, $16 \%$ for differentiated thyroid carcinoma and $22 \%$ for papillary thyroid carcinoma. In addition, high serum TSH was associated with a reduced risk for follicular thyroid carcinoma (OR $=0.73,95 \% \mathrm{CI}: 0.52,1.02)$. This meta-analysis suggested high serum TSH concentration is risky for papillary thyroid carcinoma but not for follicular thyroid carcinoma.

\section{INTRODUCTION}

Thyroid cancer is the most common malignant tumor of the endocrine system [1]. In 2003, the American Cancer Society reported an incidence of 1/10000 in the USA. Notably, the incidence of thyroid cancer is rising faster than any other malignancy [1]. Thyroid carcinoma according to the histological type can be classified as differentiated and undifferentiated. The most frequent types include papillary thyroid carcinoma (PTC), follicular thyroid carcinoma (FTC), differentiated thyroid carcinoma (DTC) [2].

Thyrotropin (TSH) is the major growth factor for thyroid cells. TSH plays a role in thyroid growth and organogenesis $[3,4]$. It has been reported that higher serum TSH concentration is associated with an increased risk of thyroid cancer [5]. TSH is thought to play roles in carcinogenesis $[6,7]$ and suppression therapy of TSH using thyroid hormone is widely used for DTC $[8,9]$. However, the risk of thyroid malignancy to serum TSH might depend on histological types. In order to address this issue, the patients with thyroid nodule and different histological thyroid cancer, including DTC, PTC as well as FTC, were included to analyze the association of thyroid cancer and serum TSH. An overall and dose-response meta-analysis were performed for this serum TSH and thyroid cancer risk by histological type.

\section{RESULTS}

Literature search and study characteristics

Of 6995 articles obtained on a literature search, 125 papers passed the screening phase with the fulltext articles reviewed (Figure 1). This yielded a final total of 22 studies [1, 7, 10-29] being combined in meta-analysis (53,538 participants). The most common reasons for study rejection included: studies did not report quantitative TSH $(n=71)$, the articles were reviews $(n=12)$ or overlapping subjects $(n=7)$. 
The duration of follow-up ranged from 1 to 20 years. Among these studies, 22 studies [1, 7, 10-29] for overall thyroid cancer, 10 studies $[1,8,15,17,19$, $21,22,24,28,29]$ for differentiated thyroid carcinoma, 3 studies [1, 15, 28] for papillary thyroid carcinoma, and 2 studies $[19,24]$ for follicular thyroid carcinoma. Main characteristics of the studies are shown in Table 1. Newcastle-Ottawa Scale (NOS) was analyzed for quality assessment and all these included studies were above 6 stars (Supplementary Table S1).

\section{Quantitative synthesis}

\section{Overall meta-analyses}

In meta-analysis for all histological thyroid cancer risk and serum TSH, 47,297 patients from 19 studies were involved. As shown in Figure 2A, the pooled OR for all histological thyroid cancer was 1.48 (95\% CI 1.23-1.79, $\mathrm{I}^{2}=94.8 \%$ ) based on the random-effects models. In metaanalysis of DTC prevalence and serum TSH, 10 studies including 43,922 patients were involved. As shown in Figure 2B, the pooled OR was 1.88 (95\% CI 1.78-1.98, $\mathrm{I}^{2}=88.3 \%$ ). In meta-analysis of PTC prevalence and serum TSH, 3 studies including 38,457 patients were involved. As shown in Figure 2C, the pooled OR was $2.08\left(95 \%\right.$ CI $\left.1.95-2.22, \mathrm{I}^{2}=87 \%\right)$. In meta-analysis of FTC prevalence and serum TSH, two studies including 1802 patients were involved. As shown in Figure 2D, high serum TSH demonstrated a reduced risk on FTC. The pooled OR for FTC patients was 0.73 (95\% CI 0.52-1.02, $\left.\mathrm{I}^{2}=0\right)$.

When comparing all histological thyroid cancer, the pooled odds ratios of thyroid cancer in patients with nodules was found to increase significantly with higher serum TSH concentrations for differentiated thyroid carcinoma (1.88vs.1.48, $P=0.0000)$ and papillary thyroid carcinoma (2.08 vs. 1.48, $P=0.0006)$ (Figure 3). On the contrary, the OR of FTC was significantly lower than all histological thyroid cancer $(0.73$ vs. $1.48, P=0.0000)$, DTC (0.73vs. $1.88, P=0.0000)$ as well as PTC (0.73vs. 2.08, $P=0.0399$ ) (Figure 3).

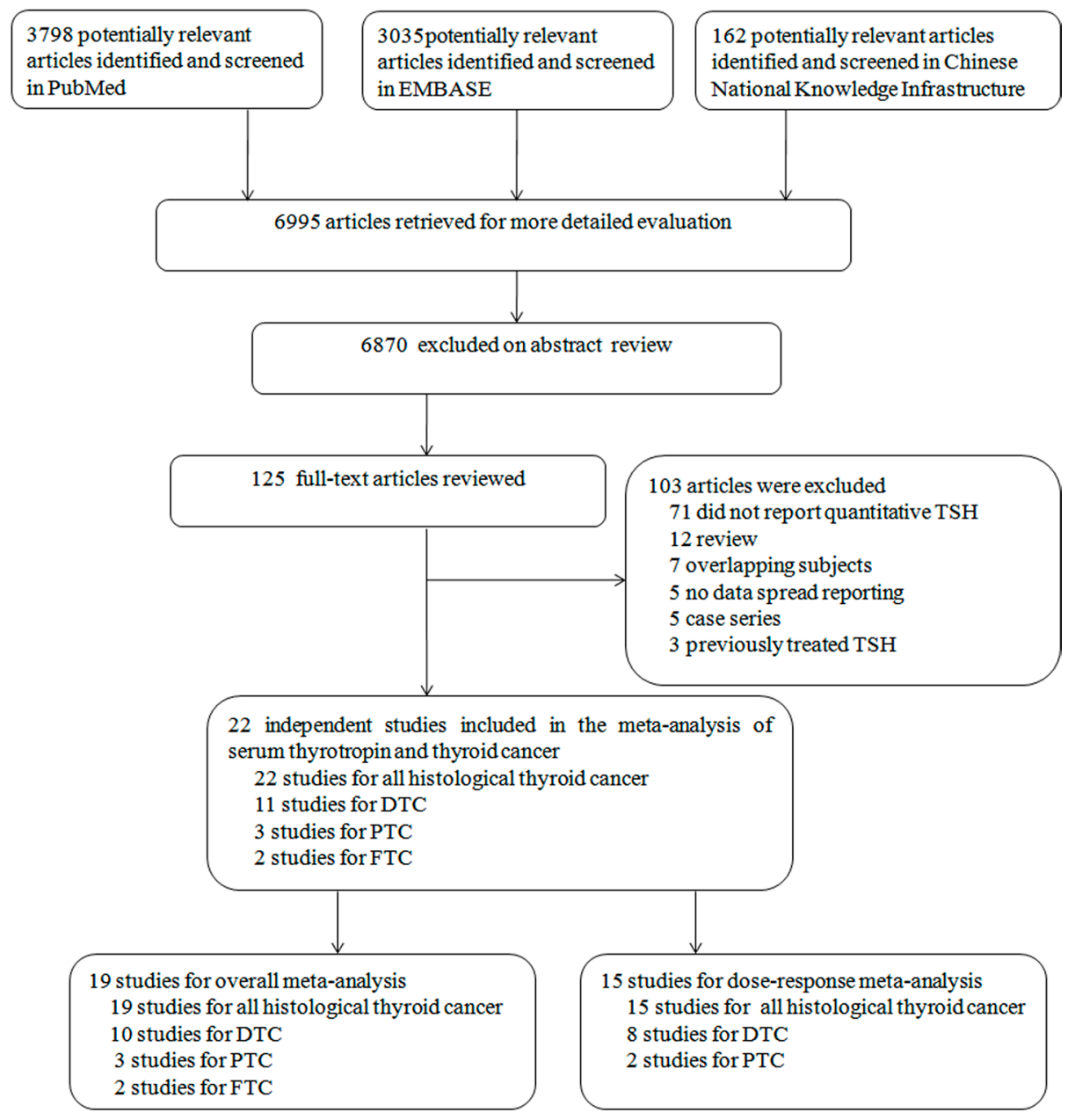

Figure 1: Flowchart of publication selection for the meta-analysis. 
Table 1: Overview of included studies

\begin{tabular}{|c|c|c|c|c|c|c|c|c|c|c|c|c|c|}
\hline $\begin{array}{l}\text { First author } \\
\text { (country, } \\
\text { year) }\end{array}$ & $\begin{array}{l}\text { Study } \\
\text { type }\end{array}$ & $\begin{array}{c}\text { Mean } \\
\text { age }\end{array}$ & $\begin{array}{l}\text { Percent } \\
\text { female } \\
\text { subject }\end{array}$ & $\begin{array}{c}\text { Numbers } \\
\text { of cases/ } \\
\text { total } \\
\text { analyzed }\end{array}$ & $\begin{array}{c}\text { Duration } \\
\text { of } \\
\text { follow-up }\end{array}$ & $\begin{array}{l}\text { Control } \\
\text { group }\end{array}$ & $\begin{array}{l}\text { Types of } \\
\text { thyroid } \\
\text { cancer } \\
\text { included } \\
(\%)\end{array}$ & $\begin{array}{c}\text { TSH } \\
\text { category } \\
\text { (mU/liter) }\end{array}$ & $\begin{array}{c}\text { OR for TSH } \\
\text { category }(95 \% \\
\text { CI) }\end{array}$ & $\begin{array}{l}\text { Covariates } \\
\text { adjusted for in } \\
\text { analysis }\end{array}$ & $\begin{array}{c}\text { Overall } \\
\text { meta- } \\
\text { analysis } \\
\text { included }\end{array}$ & $\begin{array}{l}\text { Dose- } \\
\text { response } \\
\text { analysis } \\
\text { included }\end{array}$ & $\begin{array}{c}\text { Quality } \\
\text { score }\end{array}$ \\
\hline $\begin{array}{c}\text { Boelaert et al. } \\
\text { (UK, 2006) } \\
{[10]}\end{array}$ & $\begin{array}{l}\text { Prosp. } \\
\text { cross- } \\
\text { sect }\end{array}$ & 47.8 & 86.9 & $92 / 1183$ & 18 & $\begin{array}{l}\text { Thyroid } \\
\text { nodule } \\
\text { patients }\end{array}$ & $\begin{array}{c}\text { All } \\
\text { histological } \\
\text { thyroid } \\
\text { cancer }\end{array}$ & \begin{tabular}{|l|}
$<0.4$ \\
$0.4-0.9$ \\
$1.0-1.7$ \\
$1.8-5.5$ \\
$>5.5$
\end{tabular} & \begin{tabular}{|l|}
1 \\
$1.31(0.45-3.81)$ \\
$2.72(1.02-7.27)$ \\
$3.88(1.48-10.19)$ \\
$11.18(3.23-38.63)$
\end{tabular} & $\begin{array}{c}\text { Age, goiter } \\
\text { type, and } \\
\text { serum TSH } \\
\text { concentration }\end{array}$ & Y & Y & 9 \\
\hline $\begin{array}{c}\text { Haymart et al. } \\
\text { (UK, 2008) } \\
{[11]}\end{array}$ & $\begin{array}{c}\text { Retrosp. } \\
\text { cross- } \\
\text { sect }\end{array}$ & 46 & 80.8 & $212 / 735$ & 13 & $\begin{array}{l}\text { Benign } \\
\text { surgical } \\
\text { patients }\end{array}$ & $\begin{array}{c}\text { PTC } \\
(87 \%) \text { FTC/ } \\
\text { HCC }(7 \%) \\
\text { Other }(6 \%)\end{array}$ & \begin{tabular}{|l}
$<0.06$ \\
$0.06-0.39$ \\
$0.40-1.39$ \\
$1.40-2.49$ \\
$2.50-4.99$ \\
$>5.00$
\end{tabular} & $\begin{array}{l}1 \\
1.65(0.59-4.60) \\
1.39(0.59-3.27) \\
2.50(1.04-6.04) \\
3.52(1.37-9.02) \\
4.56(1.35-15.45)\end{array}$ & $\begin{array}{l}\text { Age, nodule } \\
\text { size, and } \\
\text { preoperative } \\
\text { serum }\end{array}$ & Y & Y & 9 \\
\hline $\begin{array}{c}\text { Jonklaas et al. } \\
\text { (USA, 2008) } \\
{[12]}\end{array}$ & $\begin{array}{c}\text { Retrosp. } \\
\text { cross- } \\
\text { sect }\end{array}$ & 49 & 74 & $17 / 50$ & 3.5 & $\begin{array}{l}\text { Benign } \\
\text { surgical } \\
\text { patients }\end{array}$ & $\begin{array}{c}\text { PTC } \\
(88 \%) \text { FTC } \\
(12 \%)\end{array}$ & \begin{tabular}{|l}
$0.34-1.1$ \\
$1.2-2.1$ \\
$2.1-2.8$ \\
$>2.8$ \\
\end{tabular} & \begin{tabular}{|l}
1 \\
$8.6(2.0-35.9)$ \\
$6.0(0.6-55.7)$ \\
(No cases) \\
\end{tabular} & None & $\mathrm{N}$ & Y & 7 \\
\hline $\begin{array}{c}\text { Polyzos et al. } \\
\text { (Greece, 2008) } \\
{[13]}\end{array}$ & $\begin{array}{c}\text { Retrosp. } \\
\text { cross- } \\
\text { sect }\end{array}$ & 48.2 & 86 & $36 / 383$ & 16 & $\begin{array}{l}\text { Thyroid } \\
\text { nodule } \\
\text { patients }\end{array}$ & $\begin{array}{c}\text { PTC } \\
(69 \%) \text { FTC/ } \\
\text { HCC }(17 \%) \\
\text { Other } \\
(14 \%)\end{array}$ & \begin{tabular}{|l|}
$<0.4$ \\
$0.4-0.8$ \\
$0.9-1.4$ \\
$1.5-4.0$ \\
$>4.0$
\end{tabular} & $\begin{array}{l}1 \\
0.60(0.18-1.97) \\
0.98(0.33-2.91) \\
2.54(0.99-6.52) \\
0.63(0.07-5.49)\end{array}$ & $\begin{array}{c}\text { None (excluded } \\
\text { multivariate } \\
\text { analysis) }\end{array}$ & Y & Y & 8 \\
\hline $\begin{array}{c}\text { Fiore et al. } \\
\text { (Italy, 2009) } \\
\text { [1] }\end{array}$ & $\begin{array}{l}\text { Prosp. } \\
\text { cross- } \\
\text { sect }\end{array}$ & 49.2 & 80.3 & $\begin{array}{c}504 / \\
10178\end{array}$ & 7 & $\begin{array}{l}\text { benign } \\
\text { thyroid } \\
\text { nodular } \\
\text { disease }\end{array}$ & $\begin{array}{c}\text { PTC } \\
(100 \%)\end{array}$ & $\begin{array}{l}<0.4 \\
0.4-0.59 \\
0.6-0.89 \\
0.9-1.3 \\
1.31-3.4 \\
>3.4\end{array}$ & $\begin{array}{l}1 \\
0.80(0.51-1.27) \\
2.01(1.46-2.77) \\
2.66(1.98-3.58) \\
4.29(3.17-5.08) \\
3.50(2.10-5.83)\end{array}$ & $\begin{array}{c}\text { None (excluded } \\
\text { patients taking } \\
\text { levothyroxine ) }\end{array}$ & $\mathrm{Y}$ & Y & 7 \\
\hline $\begin{array}{c}\text { Fiore et al. } \\
\text { (Italy, 2010) } \\
\text { [15] }\end{array}$ & $\begin{array}{l}\text { Prosp. } \\
\text { cross- } \\
\text { sect }\end{array}$ & 40 & 81.2 & $\begin{array}{l}1275 / \\
27914\end{array}$ & 12 & $\begin{array}{l}\text { Thyroid } \\
\text { nodule } \\
\text { patients }\end{array}$ & $\begin{array}{c}\text { PTC } \\
(100 \%)\end{array}$ & $\begin{array}{l}<0.4 \\
0.4-0.59 \\
0.6-0.89 \\
0.9-1.3 \\
1.31-3.4 \\
>3.4\end{array}$ & \begin{tabular}{|l|}
1 \\
$1.79(1.42-2.23)$ \\
$2.72(2.24-3.3)$ \\
$3.76(3.12-4.53)$ \\
$5.32(4.45-.36)$ \\
$10.36(6.34-16.89)$
\end{tabular} & None & Y & Y & 8 \\
\hline $\begin{array}{c}\text { Gul et al. } \\
\text { (Turkey, 2010) } \\
{[17]}\end{array}$ & $\begin{array}{l}\text { Retrosp. } \\
\text { cross- } \\
\text { sect }\end{array}$ & 45.5 & 78.7 & $166 / 441$ & 3 & $\begin{array}{l}\text { Benign } \\
\text { surgical } \\
\text { patients }\end{array}$ & \begin{tabular}{|c|} 
PTC \\
$(89 \%)$ FTC/ \\
HCC $(11 \%)$ \\
\end{tabular} & \begin{tabular}{|l|}
$0.4-0.62$ \\
$0.63-1.67$ \\
$1.68-4.0$
\end{tabular} & $\begin{array}{l}1 \\
2.37(1.34-4.19) \\
5.74(3.03-10.89)\end{array}$ & $\begin{array}{l}\text { Age, gender, } \\
\text { nodule type }\end{array}$ & Y & Y & 8 \\
\hline $\begin{array}{c}\text { Dorange et al. } \\
\text { (France, 2011) } \\
{[22]}\end{array}$ & $\begin{array}{l}\text { Retrosp } \\
\text {.cross- } \\
\text { sect }\end{array}$ & 44 & 80.9 & $47 / 94$ & 20 & $\begin{array}{l}\text { Benign } \\
\text { surgical } \\
\text { disease }\end{array}$ & $\begin{array}{c}\text { PTC } \\
\text { (79\%) FTC/ } \\
\text { HCC (21\%) }\end{array}$ & $\begin{array}{l}0.1-1.0 \\
1.01-2.0 \\
2.01-4.5\end{array}$ & $\begin{array}{l}1 \\
3.43(1.37-8.57) \\
11.67(2.21-61.48)\end{array}$ & \begin{tabular}{|c|} 
Matched on \\
age, gender, \\
ethnicity, \\
method of TSH \\
measurement
\end{tabular} & $\mathrm{N}$ & Y & 9 \\
\hline $\begin{array}{c}\text { Rio et al. } \\
\text { (Brazil, 2011) } \\
{[23]}\end{array}$ & $\begin{array}{c}\text { Retrosp. } \\
\text { cross- } \\
\text { sect }\end{array}$ & 49.9 & 89 & $62 / 144$ & 2 & $\begin{array}{l}\text { Benign } \\
\text { surgical } \\
\text { patients }\end{array}$ & $\begin{array}{c}\text { PTC }(87 \%) \\
\text { FTC/HCC } \\
(7 \%) \text { Other } \\
(6 \%)\end{array}$ & $\begin{array}{l}<0.4 \\
0.4-1.39 \\
1.4-2.49 \\
2.5-4.49 \\
4.5-5.5\end{array}$ & \begin{tabular}{|l}
1 \\
$1.33(0.31-5.81)$ \\
$1.67(0.37-7.53)$ \\
$2.2(0.43-11.22)$ \\
$1.33(0.14-12.82)$
\end{tabular} & None & $\mathrm{Y}$ & Y & 7 \\
\hline $\begin{array}{c}\text { Ding et al } \\
\text { (China, 2011) } \\
{[21]}\end{array}$ & $\begin{array}{c}\text { Retrosp. } \\
\text { cross- } \\
\text { sect }\end{array}$ & 48.3 & None & 218/956 & 8 & $\begin{array}{l}\text { Benign } \\
\text { surgical } \\
\text { patients }\end{array}$ & $\begin{array}{c}\text { All } \\
\text { histological } \\
\text { thyroid } \\
\text { cancer }\end{array}$ & \begin{tabular}{|l|}
$<0.27$ \\
$0.27-1.39$ \\
$1.40-2.29$ \\
$2.23-4.20$ \\
$>4.20$
\end{tabular} & \begin{tabular}{|l}
1 \\
$0.81(0.39-1.66)$ \\
$1.01(0.49-2.06)$ \\
$1.81(0.88-3.71)$ \\
$2.03(0.82-5.03)$
\end{tabular} & None & Y & Y & 7 \\
\hline $\begin{array}{c}\text { Zafon et al. } \\
\text { (Spain, 2012) } \\
{[26]}\end{array}$ & $\begin{array}{l}\text { Retrosp. } \\
\text { cross- } \\
\text { sect }\end{array}$ & 53.8 & 80.9 & $76 / 386$ & 3 & $\begin{array}{l}\text { Benign } \\
\text { surgical } \\
\text { patients }\end{array}$ & $\begin{array}{c}\text { PTC }(96 \%) \\
\text { FTC }(4 \%)\end{array}$ & \begin{tabular}{|l|}
$<0.4$ \\
$0.4-4$ \\
$>4$
\end{tabular} & $\begin{array}{l}1 \\
1.91(0.93-3.92) \\
5.38(1.75-16.58)\end{array}$ & None & Y & Y & 6 \\
\hline $\begin{array}{c}\text { Kim et al. } \\
\text { (Korea, 2010) } \\
{[19]}\end{array}$ & $\begin{array}{c}\text { Retrosp. } \\
\text { cross- } \\
\text { sect }\end{array}$ & 50.9 & 75.8 & $296 / 1638$ & 2 & $\begin{array}{l}\text { benign } \\
\text { thyroid } \\
\text { nodular } \\
\text { disease }\end{array}$ & $\begin{array}{l}\text { PTC }(99 \%) \\
\text { Other (1\%) }\end{array}$ & $\begin{array}{l}<0.17 \\
0.17-1.17 \\
1.18-2.01 \\
2.02-4.05 \\
>4.06\end{array}$ & $\begin{array}{l}1 \\
0.99(0.27-3.63) \\
1.44(0.93-2.23) \\
1.72(1.12-2.63) \\
1.98(1.06-3.70)\end{array}$ & $\begin{array}{l}\text { Age, gender, } \\
\text { nodule size, } \\
\text { nodule type, } \\
\text { thyroid } \\
\text { autoimmunity }\end{array}$ & Y & Y & 8 \\
\hline $\begin{array}{c}\text { Haymart et al. } \\
\text { (USA, 2009) } \\
{[14]}\end{array}$ & $\begin{array}{c}\text { Retrosp. } \\
\text { cross- } \\
\text { sect }\end{array}$ & 48.9 & 80.0 & $212 / 735$ & 13 & $\begin{array}{l}\text { Benign } \\
\text { surgical } \\
\text { patients }\end{array}$ & $\begin{array}{c}\text { PTC }(52 \%) \\
\text { FTC/HCC } \\
(37 \%) \\
\text { Other } \\
(11 \%)\end{array}$ & $\begin{array}{l}<0.06 \\
0.06-0.39 \\
0.4-1.39 \\
1.4-2.49 \\
2.5-4.99 \\
>=5.0\end{array}$ & $\begin{array}{l}1 \\
1.65(0.59-4.6) \\
1.39(0.59-3.27) \\
2.5(1.04-6.04) \\
3.52(1.37-9.02) \\
4.56(1.35-15.45)\end{array}$ & $\begin{array}{l}\text { Gender, age, } \\
\text { nodule } \\
\text { size, and } \\
\text { preoperative } \\
\text { serum TSH } \\
\text { concentration }\end{array}$ & $\mathrm{N}$ & Y & 8 \\
\hline
\end{tabular}




\begin{tabular}{|c|c|c|c|c|c|c|c|c|c|c|c|c|c|}
\hline $\begin{array}{c}\text { Kim et al. } \\
\text { (Korea, 2013) } \\
{[27]}\end{array}$ & $\begin{array}{l}\text { Prosp. } \\
\text { cross- } \\
\text { sect }\end{array}$ & 47.1 & 81.7 & $\begin{array}{l}2184 / \\
3905\end{array}$ & 7 & $\begin{array}{l}\text { healthy } \\
\text { controls }\end{array}$ & $\begin{array}{c}\text { PTC } \\
(96.6 \%) \\
\text { FTC }(1.4 \%) \\
\text { Other }(2 \%)\end{array}$ & $\begin{array}{l}0.40 \leq 1.10 \\
1.11 \leq 1.63 \\
1.64 \leq 2.30 \\
2.31 \leq 4.80\end{array}$ & $\begin{array}{l}1 \\
1.27(1.03-1.57) \\
1.55(1.25-1.92) \\
2.21(1.78-2.74)\end{array}$ & $\begin{array}{l}\text { Age, sex, and } \\
\text { the presence of } \\
\text { a family history } \\
\text { of thyroid } \\
\text { cancer }\end{array}$ & $\mathrm{N}$ & $\mathrm{Y}$ & 9 \\
\hline $\begin{array}{c}\text { Jin et al. (USA, } \\
\text { 2010) [18] }\end{array}$ & $\begin{array}{c}\text { Retrosp. } \\
\text { cross- } \\
\text { sect }\end{array}$ & 49 & 86 & $135 / 660$ & 18 & $\begin{array}{l}\text { Thyroid } \\
\text { Nodule } \\
\text { patients }\end{array}$ & $\begin{array}{l}\text { PTC }(87 \%) \\
\text { FTC }(9 \%) \\
\text { Other }(4 \%)\end{array}$ & $\begin{array}{l}<0.9 \\
0.9-1.7 \\
1.8-5.5 \\
>5.5\end{array}$ & $\begin{array}{l}1 \\
2.05(1.25-3.35) \\
2.36(1.31-4.25) \\
6.17(1.77-21.46)\end{array}$ & Age and sex & $\mathrm{Y}$ & $\mathrm{Y}$ & 7 \\
\hline $\begin{array}{c}\text { Kim et al. } \\
\text { (Korea, 2012) } \\
{[7]}\end{array}$ & $\begin{array}{l}\text { Retrosp. } \\
\text { cross- } \\
\text { sect }\end{array}$ & 48.2 & 80.1 & $\begin{array}{c}52 / \\
1329\end{array}$ & 4 & $\begin{array}{l}\text { Benign } \\
\text { surgical } \\
\text { patients }\end{array}$ & $\begin{array}{c}\text { PTC }(98 \%) \\
\text { FTC }(2 \%)\end{array}$ & Continuous & $0.70(0.47-1.03)$ & None & $\mathrm{Y}$ & $\mathrm{N}$ & 8 \\
\hline $\begin{array}{c}\text { Lee et } \\
\text { al.(Korea, } \\
2012) \\
{[24]}\end{array}$ & $\begin{array}{c}\text { Retrosp. } \\
\text { cross- } \\
\text { sect }\end{array}$ & None & None & $35 / 164$ & 7 & $\begin{array}{l}\text { Benign } \\
\text { surgical } \\
\text { patients }\end{array}$ & $\begin{array}{l}\text { PTC }(54 \%) \\
\text { FTC }(46 \%)\end{array}$ & Continuous & $\begin{array}{l}0.804(0.410- \\
1.575)\end{array}$ & None & $\mathrm{Y}$ & $\mathrm{N}$ & 7 \\
\hline $\begin{array}{c}\text { Jiao et al } \\
\text {.(China, 2015) } \\
{[28]}\end{array}$ & $\begin{array}{c}\text { Retrosp. } \\
\text { cross- } \\
\text { sect }\end{array}$ & 49.2 & 75.6 & $113 / 365$ & 2 & $\begin{array}{l}\text { Benign } \\
\text { surgical } \\
\text { patients }\end{array}$ & $\begin{array}{c}\text { PTC } \\
(100 \%)\end{array}$ & Continuous & $1.52(1.01-2.42)$ & None & $\mathrm{Y}$ & $\mathrm{N}$ & 7 \\
\hline $\begin{array}{c}\text { Nixon et al. } \\
\text { (USA, 2010) } \\
{[20]}\end{array}$ & $\begin{array}{c}\text { Retrosp. } \\
\text { cross- } \\
\text { sect. }\end{array}$ & 55 & 75.0 & $111 / 156$ & 1 & $\begin{array}{l}\text { Thyroid } \\
\text { Nodule } \\
\text { patients }\end{array}$ & $\begin{array}{c}\text { All } \\
\text { histological } \\
\text { thyroid } \\
\text { cancer }\end{array}$ & Continuous & $3.53(1.35-9.24)$ & None & $\mathrm{Y}$ & $\mathrm{N}$ & 8 \\
\hline $\begin{array}{c}\text { Moon et al. } \\
\text { (Korea, 2012) } \\
{[25]}\end{array}$ & $\begin{array}{l}\text { Retrosp. } \\
\text { cross- } \\
\text { sect }\end{array}$ & 53.5 & 84.1 & $42 / 483$ & 1 & $\begin{array}{l}\text { Thyroid } \\
\text { Nodule } \\
\text { patients }\end{array}$ & $\begin{array}{c}\text { All } \\
\text { histological } \\
\text { thyroid } \\
\text { cancer } \\
\end{array}$ & Continuous & $\begin{array}{l}1.402(1.018- \\
1.932)\end{array}$ & $\begin{array}{l}\text { None(exclude } \\
\text { multivariate } \\
\text { analysis) }\end{array}$ & $\mathrm{Y}$ & $\mathrm{N}$ & 9 \\
\hline $\begin{array}{c}\text { Maia et al. } \\
\text { (Brazil, 2011) } \\
{[7]}\end{array}$ & $\begin{array}{c}\text { Retrosp. } \\
\text { Cross- } \\
\text { sect. }\end{array}$ & 47.2 & 84.6 & $50 / 143$ & 10 & $\begin{array}{l}\text { Benign } \\
\text { surgical } \\
\text { patients }\end{array}$ & $\begin{array}{c}\text { All } \\
\text { histological } \\
\text { thyroid } \\
\text { cancer } \\
\end{array}$ & Continuous & $1.03(0.97-1.08)$ & $\begin{array}{l}\text { Age, gender, } \\
\text { nodule type }\end{array}$ & $\mathrm{Y}$ & $\mathrm{N}$ & 8 \\
\hline $\begin{array}{l}\text { Gerschpacher } \\
\text { et al. (Austria, } \\
\text { 2010) [16] }\end{array}$ & $\begin{array}{c}\text { Retrosp. } \\
\text { cross- } \\
\text { sect }\end{array}$ & 55 & 44.8 & $33 / 87$ & 14 & $\begin{array}{c}\text { Medullary } \\
\text { cancer, C } \\
\text { cell } \\
\text { hyperplasia }\end{array}$ & $\begin{array}{c}\text { All } \\
\text { histological } \\
\text { thyroid } \\
\text { cancer }\end{array}$ & Continuous & $0.86(0.58-1.25)$ & Age, gender & $\mathrm{Y}$ & $\mathrm{N}$ & 8 \\
\hline
\end{tabular}

Prosp.cross-sect, prospective cross-sectional; Retrosp. cross-sect, retrospective cross-sectional; OR, odds ratio; N/A, not available; CI, confidence interval; Y, included; N, not included.

\section{Dose-response meta-analysis}

15 studies involving 50,811 participants were included in the dose-response meta-analysis of all histological thyroid cancer risk and serum TSH. And a nonlinear relationship was found $(p=0.000)$ as shown in Figure 4A. Compared with benign thyroid disease patients, the fractional polynomial model estimates of the OR were 1.4 and 1.6 for 2.0 and $4.0 \mathrm{mU} / \mathrm{L}$ of serum TSH respectively. This meta-analysis showed a $14 \%$ increase of thyroid cancer risk for each $1 \mathrm{mU} / \mathrm{L}$ increase in serum TSH.

8 studies involving 43,675 participants were included in the dose-response meta-analysis of serum TSH and DTC. And a nonlinear relationship was found $(p=0.000)$ as shown in Figure 4B. Compared with benign thyroid disease patients, the fractional polynomial model estimates of the OR were 1.7 and 1.8 for 2.0 and $4.0 \mathrm{mU} / \mathrm{L}$ of serum TSH respectively. This meta-analysis showed a $16 \%$ increase of DTC risk for each $1 \mathrm{mU} / \mathrm{L}$ increase in serum TSH.

2 studies involving 38,092 participants were included in the dose-response meta-analysis of the serum TSH and PTC. And a nonlinear relationship was found $(p=0.000)$ as shown in Figure 4C. Compared with benign thyroid disease patients, the fractional polynomial model estimates of the OR were 1.9 and 2.0 for 2.0 and $4.0 \mathrm{mU} / \mathrm{L}$ of serum TSH respectively. This meta-analysis showed a $22 \%$ increase of PTC risk for each $1 \mathrm{mU} / \mathrm{L}$ increase in serum TSH.

\section{Meta-regression analyses}

We conducted the meta-regression analysis for the evaluation the heterogeneity to investigate whether the association between TSH and thyroid carcinoma was modified by patient source, publication year and study type. We found that the study type can explain $45.08 \%$ heterogeneity. The overall OR of thyroid cancer calculated from prospective studies was 1.95 (95\% CI 1.59-2.39). However, the corresponding OR from retrospective studies was 1.39 (95\% CI 1.18-1.63), which was significantly lower than the former one $(P<0.001)$.

\section{Sensitivity analysis}

In a sensitivity analysis in which one study at a time was removed and the rest analyzed, the pooled OR ranged from 1.93 to 2.08 for overall thyroid carcinoma, which indicated that the pooled estimates were stable and not influenced by a single study (Supplementary Table S2). 
A

Study ID

Boelaert et al. 2006

Haymart et al.2007

Polyzos et al. 2008

Fiore et al. 2009

Fiore et al. 2010

Gul et al. 2010

Kim et al.2010

Ding et al.2011

Rio et al. 2011

Kim et al. 2012

Zafon et al. 2012

lee et al.2012

Zafon et al. 2014

Nixon et al.2014

Jiao et al.2015

Jin et a. 2010

Moon et al.2012

Maia et al.2010

Gerschpacher et al. 2010

Overall (l-squared $=94.8 \%, p=0.000$ )

NOTE: Weights are from random effects analysis

0.2

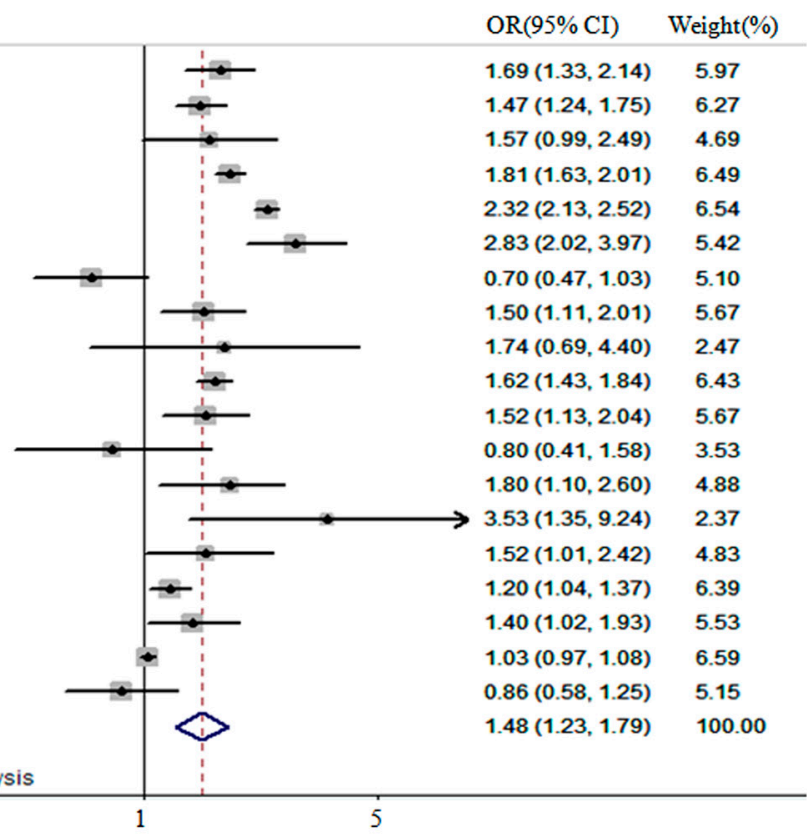

B

Study ID

OR $(95 \% \mathrm{CI}) \quad$ Weight $(\%)$

Haymart et al.2007

Fiore et al. 2009

Fiore et al. 2010

Gul et al. 2010

Kim et al.2010

Dorange et al.2011

Ding et al.2011

Kim et al. 2012

lee et al.2012

Jiao et al.2015

Overall (I-squared $=88.3 \%, p=0.000)$

$1.47(1.24,1.75) 9.22$

$1.81(1.63,2.01) \quad 25.79$

$2.32(2.13,2.52) 38.63$

$2.83(2.02,3.97) \quad 2.37$

$0.70(0.47,1.03) 1.76$

$1.30(0.20,4.40) 0.11$

$1.50(1.11,2.01) \quad 3.14$

$1.62(1.43,1.84) \quad 16.95$

$0.80(0.41,1.58) \quad 0.60$

$1.52(1.01,2.42) \quad 1.42$

$1.88(1.78,1.98) \quad 100.00$

\begin{tabular}{ll|l|l}
\hline 0.2 & 1 & 5
\end{tabular}

C

Study ID

OR $(95 \% \mathrm{CI}) \quad$ Weight $(\%)$

Fiore et al. 2009

Fiore et al. 2010

Jiao et al.2015

Overall (I-squared $=87.0 \%, p=0.000)$
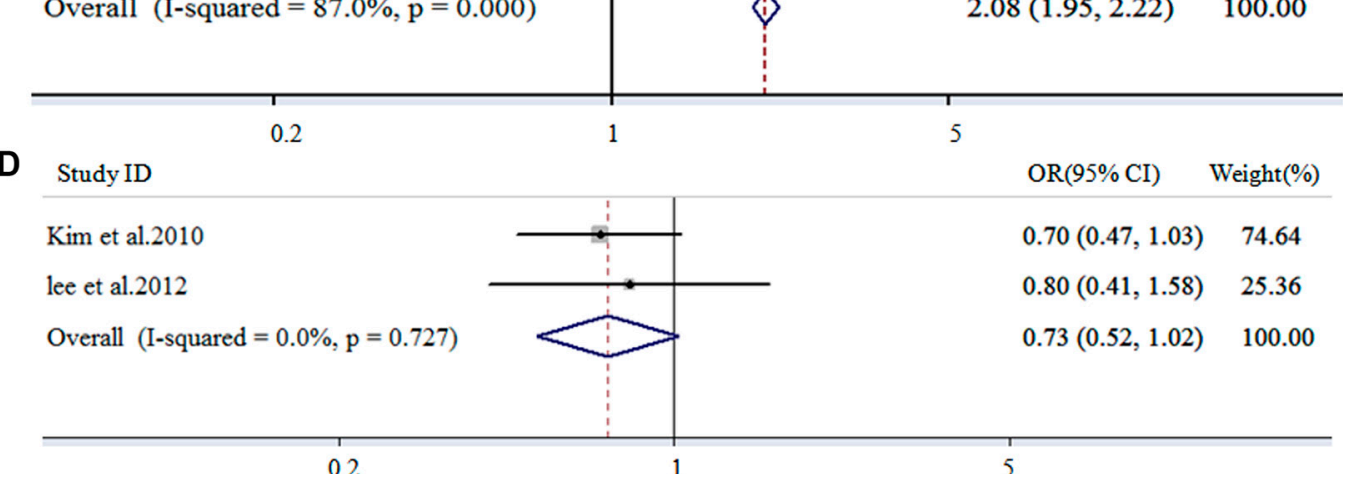

Figure 2: Forest plot for study-specific and pooled OR in overall meta-analysis. The size of each grey square is proportional to the study's weight calculated as inverse of variance. OR, odd ratio; 95\% CI, 95\% confidence intervals; thyroid cancer, all the histological types of thyroid cancer (19 studies); DTC, differentiated thyroid carcinoma (10 studies); PTC, papillary thyroid carcinoma (3 studies); FTC, follicular thyroid carcinoma (2 studies). Weights are from random effects analysis. 


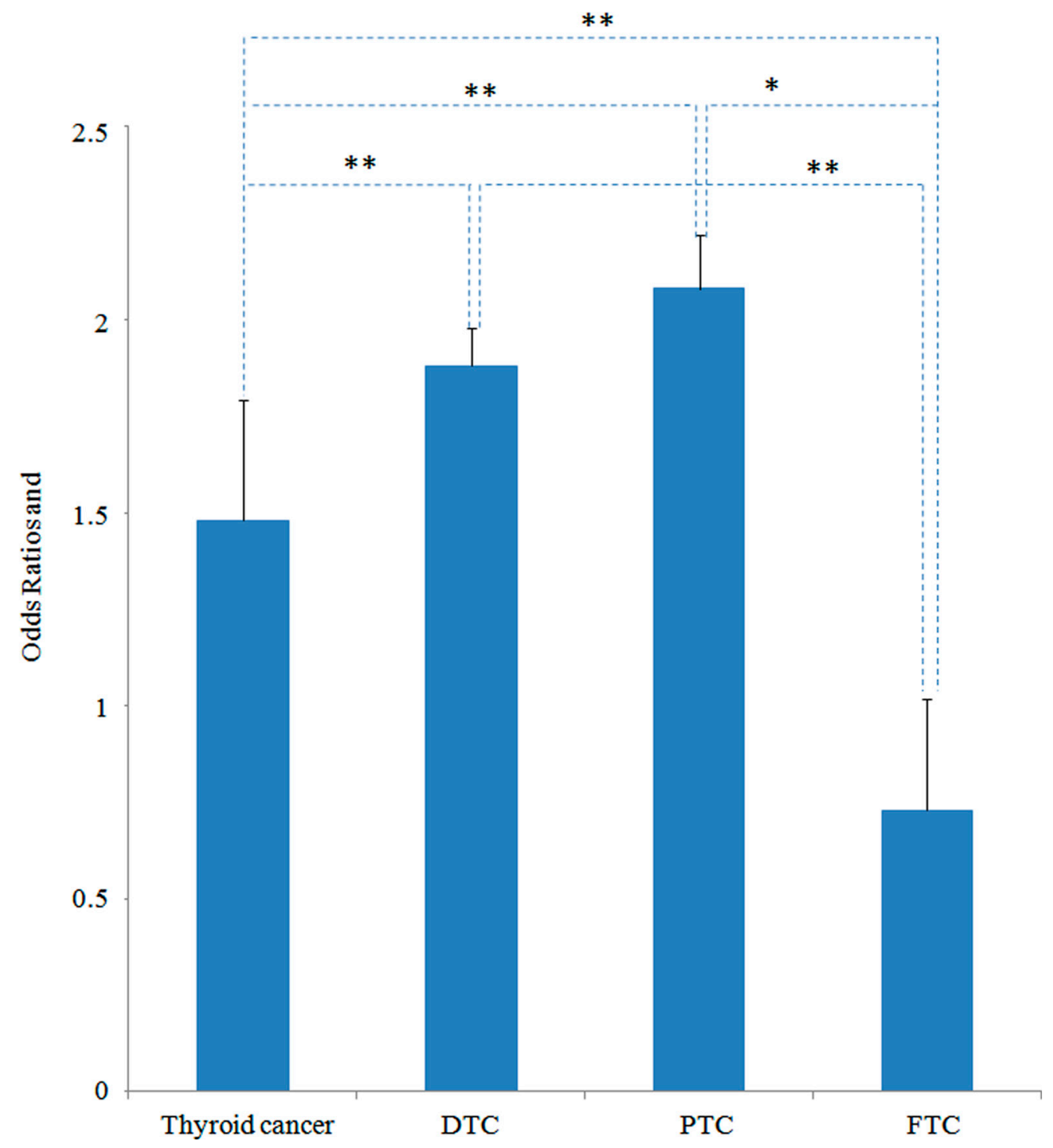

Figure 3: OR comparison between all histological thyroid cancer (thyroid cancer), differentiated thyroid carcinoma (DTC), papillary thyroid carcinoma (PTC) and follicular thyroid carcinoma (FTC). $* P<0.05, * * P<0.01$.
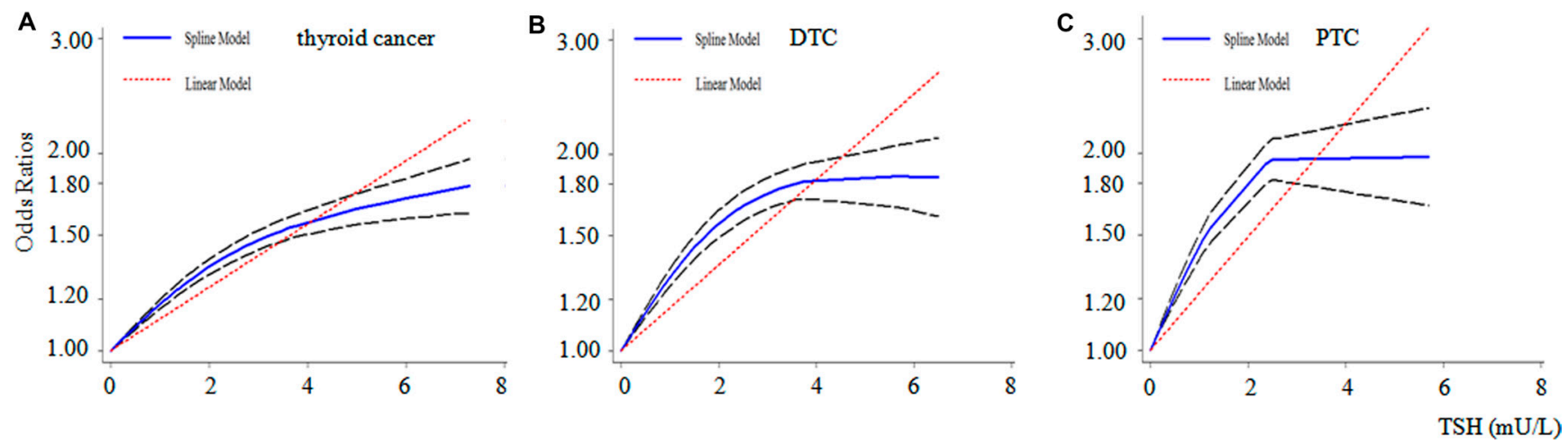

Figure 4: Dose-response relationship for serum TSH and thyroid cancer. Dotted lines represent the $95 \%$ confidence intervals for the fitted trend. The dose-response relationship plot between TSH levels (mU/L) and different histological types of thyroid cancer. Thyroid cancer, all thyroid cancer (15 studies); DTC, differentiated thyroid carcinoma (8 studies); PTC, papillary thyroid carcinoma (2 studies). 


\section{Publication bias}

No evidence for publication bias was indicated by Egger's regression test $(P=0.448)$. The funnel plot also indicated no evidence of publication bias.

\section{DISCUSSION}

This study provided a new view for a potential relation between serum TSH and risk of thyroid cancer by overall meta-analysis and dose-response meta-analysis. This study indicated that the risk of thyroid cancer for higher serum TSH depends on histological types. Higher serum TSH was revealed to be associated with increased risk for PTC but reduced risk for FTC. Given the large number and the high prevalence of thyroid cancer worldwide [1,12], the results from this meta-analysis have important implications. However, our result for serum TSH and FTC was based on two pre-operation studies. It needs more studies for verification.

In addition, FTC belongs to DTC and TSH suppression was widely used after DTC thyroidectomy $[8,9,14]$. Even that, this meta-analysis suggested the higher serum TSH would lead to reduced risk for FTC. TSH effect on FTC with TSH suppression treatment after operation might be different and random clinical trials for the TSH treatment on FTC would present direct evidence. In addition, further biological experiments are needed to verify this difference between PTC and FTC and reveal the molecular mechanisms under the difference. For example, the expression of TSH receptor in most DTC thyroid cancers is similar to or slightly lower than that found in normal thyrocytes. And TSH was thought promote cancer cell proliferation via its receptors [11]. However, it has been reported that the proliferation of a FTC cell line without TSH receptor expression was inhibited after ectopic expression of TSH receptor, which indicated the effect of TSH on DTC may be complicated and depends on the cell context [30].

Our meta-analysis had several strengths, including the associations for differentiated thyroid carcinoma, papillary thyroid carcinoma and follicular thyroid cancer were evaluated by categories of thyroid cancer separately. In addition, two different methods of overall and doseresponse meta-analysis were used to investigate the association between thyroid cancer risk and serum TSH, which presented both the pooled and dynamic view of their relationship.

Our meta-analysis also had some limitations. A statistically significant heterogeneity between the studies was observed, which was likely to be attributed to the variation in study design. The relatively small number of studies limited our ability to identify other histological groups. Unpublished data, non-English-language studies, and missed studies may exist and may have influenced our results. Furthermore, more studies are needed on cancer stage, grade, metastasis and morbidity rate to quantify with greater confidence the nature of the relationship between TSH and risk of subgroup PTC.

In conclusion, findings from this meta-analysis of studies suggested that the risk of thyroid cancer for higher serum TSH depends on histological types. Higher serum TSH concentration is associated with increased risk of PTC but reduced risk of FTC.

\section{MATERIALS AND METHODS}

\section{Search strategy}

We conducted a systematic literature search using the PUBMED, EMBASE and Chinese National Knowledge Infrastructure databases to April 21, 2016. The search strategy in these databases all comprised MeSH terms of "thyroid neoplasm" or "thyroid cancer," as well as (all fields) "thyrotropic hormone," "thyrotropin," "TSH," and "hormothyrin," following the MOOSE (Metaanalyses Of Observational Studies) guidelines [20]. No language restriction was applied. We also hand searched reference lists from eligible papers as well as reference lists from national and international thyroid cancer and nodule guidelines [31-35].

\section{Inclusion criteria}

The criteria for inclusion were as follows: (i) observational epidemiological studies (case-control, case-cohort, or cohort) on total preoperative serum TSH levels and thyroid cancer incidence in population; (ii) the exposure was serum TSH concentration; (iii) reporting the odds ratio (OR) or relative risk (RR) estimates with the corresponding 95\% confidence intervals (CI) or sufficient information to calculate them for each 1 $\mathrm{mU} / \mathrm{L}$ increase in serum TSH; (IV) for those reports that included the same subjects (or overlapping subjects) as another study, the most informative study was chosen for the primary analysis. We excluded review articles, case reports and case series containing only thyroid cancer patients. Two investigators (NH and ZML) independently identified the eligible studies. Initially the article titles and abstracts were screened for potentially relevant papers, followed by the full-text review of the remaining studies (Figure 1). Discrepancies were resolved through consensus.

\section{Data extraction}

$\mathrm{NH}, \mathrm{ZML}$, JFL independently extracted the study data and any disagreements were resolved by consensus. Reported effect measures and confidence intervals within TSH exposure categories were extracted. Where several effect measures were reported in an article, the most completely adjusted models accounting for possible confounding was chosen. The following information was 
also extracted from each of the eligible publications: first author's name, publication year, study location, followup years, age, TSH exposure categories, ascertainment of benign thyroid disease, procedures, percentage of females.

\section{Study quality score}

Newcastle-Ottawa Scale (NOS) was analyzed for quality assessment [36] (Supplementary Table S1). The NOS awards a maximum of nine points to each casecontrol study: four for the quality of selection (adequate case definition, representativeness of cases, selection of controls, definition of controls), two for comparability (confounding) and three for the quality of the exposure (ascertainment of exposure, same method of ascertainment of cases and controls). It awards a maximum of nine points to each cohort study: four for the quality of selection (representativeness, selection of non-exposed cohort, ascertainment of exposure, no disease at start of study), two for comparability (confounding) and three for the quality of the outcome (assessment of outcome, length of follow-up and adequacy of follow-up). Studies with NOS values of six or greater were considered moderate to highquality studies and those with a NOS value of less than six were regarded low-quality studies.

\section{Statistical analysis}

Statistical analysis was performed with STATA 12.0 (Stata-Corp, College Station, TX), using twosided hypothesis testing and alpha $=0.05$. We assessed heterogeneity for both within- and between studies with the $\mathrm{I}^{2}$ statistic [37] as a measure of the proportion of total variation in estimates that is due to heterogeneity, where $\mathrm{I}^{2}$ values of $25 \%, 50 \%$, and $75 \%$ correspond to cut-off points for low, moderate, and high degrees of heterogeneity [37]. We calculated pooled ORs using the random-effects models of DerSimonian and Laird for the high degree of heterogeneity [38]. Dose-response metaanalyses were conducted by using the GLST command with the generalized least-squares method for trend estimation of summarized dose-response data, based on the Greenland and Longnecker method [39]. Restricted cubic splines were used to assess for potential curvilinear relations.

Using meta-regression analysis, we further investigated whether the association between TSH and thyroid cancer risk by histological type was modified by study-specific factors, including patient source, publication year and study type. We conducted a sensitivity analysis, in which one study at a time was removed and the rest analyzed to assess whether the results were markedly affected by a single study with STATA 12.0. Evidence of publication bias was assessed by visual inspection of funnel plots using Egger's regression test [40].

\section{ACKNOWLEDGMENTS AND FUNDING}

This work is supported by grants from National Natural Science Foundation of China (81472758, 31170783, U1302225).

\section{CONFLICTS OF INTEREST}

No conflicts of interest was declared.

\section{REFERENCES}

1. Fiore E, Rago T, Provenzale MA, Scutari M, Ugolini C, Basolo F, Di Coscio G, Berti P, Grasso L, Elisei R, Pinchera A, Vitti P. Lower levels of TSH are associated with a lower risk of papillary thyroid cancer in patients with thyroid nodular disease: thyroid autonomy may play a protective role. Endocr Relat Cancer. 2009; 16:1251-1260.

2. Xing M. Molecular pathogenesis and mechanisms of thyroid cancer. Nat Rev Cancer. 2013; 13:184-199.

3. Roger P, Taton M, Van Sande J, Dumont JE. Mitogenic effects of thyrotropin and adenosine $3^{\prime}, 5^{\prime}$-monophosphate in differentiated normal human thyroid cells in vitro. J Clin Endocrinol Metab. 1988; 66:1158-1165.

4. Derwahl M, Derwahl M. Thyrotropin May Not Be the Dominant Growth Factor in Benign and Malignant Thyroid Tumors. J Clin Endocrinol Metab. 1999; 84:829-834.

5. Mcleod DS, Watters KF, Carpenter AD, Ladenson PW, Cooper DS, Ding EL. Thyrotropin and thyroid cancer diagnosis: a systematic review and dose-response metaanalysis. J Clin Endocrinol Metab. 2012; 97:2682-2692.

6. Chia SY, Milas M, Reddy SK, Siperstein A, Skugor M, Brainard J, Gupta MK. Thyroid-stimulating hormone receptor messenger ribonucleic acid measurement in blood as a marker for circulating thyroid cancer cells and its role in the preoperative diagnosis of thyroid cancer. J Clin Endocrinol Metab. 2007; 92:468-475.

7. Maia FF, Matos PS, Silva BP, Pallone AT, Pavin EJ, Vassallo J, Zantut-Wittmann DE. Role of ultrasound, clinical and scintigraphyc parameters to predict malignancy in thyroid nodule. Head Neck Oncol. 2011; 3:23-30.

8. Brabant G. Thyrotropin suppressive therapy in thyroid carcinoma: what are the targets? J Clin Endocrinol Metab J Clin Endocrinol Metab. 2008; 93:1167-1169.

9. Davis PJ, Hercbergs A, Luidens MK, Lin HY. Recurrence of differentiated thyroid carcinoma during full TSH suppression: is the tumor now thyroid hormone dependent? Horm Cancer. 2015; 6:7-12.

10. Boelaert K, Horacek J, Holder RL, Watkinson JC, Sheppard MC, Franklyn JA. Serum thyrotropin concentration as a novel predictor of malignancy in thyroid nodules investigated by fine-needle aspiration. J Clin Endocrinol Metab. 2006; 91:4295-4301.

11. Haymart MR, Repplinger DJ, Leverson GE, Elson DF, Sippel RS, Jaume JC, Chen H. Higher serum thyroid stimulating 
hormone level in thyroid nodule patients is associated with greater risks of differentiated thyroid cancer and advanced tumor stage. J Clin Endocrinol Metab. 2008; 93:809-814.

12. Jonklaas J, Nsouli-Maktabi H, Soldin SJ. Endogenous thyrotropin and triiodothyronine concentrations in individuals with thyroid cancer. Thyroid. 2008; 18:943-952.

13. Polyzos SA, Kita M, Efstathiadou Z, Poulakos P, Slavakis A, Sofianou D, Flaris N, Leontsini M, Kourtis A, Avramidis A. Serum thyrotropin concentration as a biochemical predictor of thyroid malignancy in patients presenting with thyroid nodules. J Cancer Res Clin Oncol. 2008; 134:953-960.

14. Haymart MR, Glinberg SL, Liu J, Sippel RS, Jaume JC, Chen H. Higher serum TSH in thyroid cancer patients occurs independent of age and correlates with extrathyroidal extension. Clin Endocrinol (Oxf). 2009; 71:434-439.

15. Fiore E, Rago T, Provenzale MA, Scutari M, Ugolini C, Basolo F, Di Coscio G, Miccoli P, Grasso L, Pinchera A, Vitti P. L-thyroxine-treated patients with nodular goiter have lower serum TSH and lower frequency of papillary thyroid cancer: results of a cross-sectional study on 27914 patients. Endocr Relat Cancer. 2010; 17:231-239.

16. Gerschpacher M, Göbl C, Anderwald C, Gessl A, Krebs M. Thyrotropin serum concentrations in patients with papillary thyroid microcancers. Thyroid. 2010; 20:389-392.

17. Gul K, Ozdemir D, Dirikoc A, Oguz A, Tuzun D, Baser H, Ersoy R, Cakir B. Are endogenously lower serum thyroid hormones new predictors for thyroid malignancy in addition to higher serum thyrotropin? Endocrine. 2010; 37:253-260.

18. Jin J, Machekano R, Mchenry CR. The utility of preoperative serum thyroid-stimulating hormone level for predicting malignant nodular thyroid disease. Am J Surg. 2010; 199:294-298.

19. Kim ES, Lim DJ, Baek KH, Lee JM, Kim MK, Kwon HS, Song KH, Kang MI, Cha BY, Lee KW, Son HY. Thyroglobulin antibody is associated with increased cancer risk in thyroid nodules. Thyroid. 2010; 20:885-891.

20. Nixon IJ, Ian G, Hann LE, Oscar L, Changhong Y, Suzanne B, Shah JP, Ashok S, Kattan MW, Patel SG. Nomogram for predicting malignancy in thyroid nodules using clinical, biochemical, ultrasonographic, and cytologic features. Surgery. 2010; 148:1120-1127.

21. Ding W. Clinical significance of thyrotropin in differentiated thyroid cancer. Shandong: Univ. of shandong 2011.

22. Dorange A, Triau S, Mucci-Hennekinne S, Bizon A, Laboureau-Soares S, Illouz F, Rodien P, Rohmer V. An elevated level of TSH might be predictive of differentiated thyroid cancer. Ann Endocrinol(Paris). 2011; 72:513-521.

23. Rio ALS, Biscolla RPM, Andreoni DM, Camacho CP, Ikejiri ES, Matsumura LK, Hidal JT, Maciel RMDB. Evaluation of clinical, laboratorial and ultrasonographic predicting factors of malignancy in thyroid nodules. Arq Bras Endocrinol Metabol. 2011; 55:29-37.

24. Lee EK, Chung KW, Min HS, Kim TS, Kim TH, Ryu JS, Jung YS, Kim SK, Lee YJ. Preoperative serum thyroglobulin as a useful predictive marker to differentiate follicular thyroid cancer from benign nodules in indeterminate nodules. J Korean Med Sci. 2012; 27:1014-1018.

25. Moon SS, Lee YS, Lee IK, Kim JG. Serum thyrotropin as a risk factor for thyroid malignancy in euthyroid subjects with thyroid micronodule. Head Neck. 2012; 34:949-952.

26. Zafon C, Obiols G, Baena JA, Castellvi J, Dalama B, Mesa J. Preoperative thyrotropin serum concentrations gradually increase from benign thyroid nodules to papillary thyroid microcarcinomas then to papillary thyroid cancers of larger size. J Thyroid Res. 2012; 2012:530721.

27. Kim HK, Yoon JH, Kim SJ, Cho JS, Kweon SS, Kang HC. Higher TSH level is a risk factor for differentiated thyroid cancer. Clin Endocrinol (Oxf). 2013; 78:472-477.

28. Zhou JjYS. Relationship between serum thyroxin-stimulating hormone and papillary thyroid micrcarcinoma in nodular thyroid disease. Zhonghua Yi Xue Za Zhi. 2015; 95:908-911.

29. Kim KW, Park YJ, Rn EHK, Park SY, Park DJ, Ahn SH, Park DJ, Jang HC, Bo YC. Elevated risk of papillary thyroid cancer in Korean patients with Hashimoto's thyroiditis. Head \& Neck. 2011; 33:691-695.

30. Hoelting T, Duh QY, Clark OH, Herfarth C. Transfection of follicular thyroid gland carcinoma cells with human TSH receptor changes growth, invasion and adhesion Langenbecks Arch Chir Suppl Kongressbd. 1998; 115:281-284.

31. American Thyroid Association Guidelines Taskforce on Thyroid N, Differentiated Thyroid C, Cooper DS, Doherty GM, Haugen BR, Kloos RT, Lee SL, Mandel SJ, Mazzaferri EL, McIver B, Pacini F, Schlumberger M, Sherman SI, et al. Revised American Thyroid Association management guidelines for patients with thyroid nodules and differentiated thyroid cancer. Thyroid. 2009; 19:1167-1214.

32. Gharib H, Papini E, Paschke R, Duick DS, Valcavi R, Hegedus L, Vitti P, Nodules AAETFoT. American Association of Clinical Endocrinologists, Associazione Medici Endocrinologi, and European Thyroid Association medical guidelines for clinical practice for the diagnosis and management of thyroid nodules. J Endocrinol Invest. 2010; 33:1-50.

33. Tuttle RM, Ball DW, Byrd D, Dilawari RA, Doherty GM, Duh QY, Ehya H, Farrar WB, Haddad RI, Kandeel F, Kloos RT, Kopp P, Lamonica DM, et al. Thyroid carcinoma. J Natl Compr Canc Netw. 2010; 8:1228-1274.

34. Kendall-Taylor. Guidelines for the management of thyroid cancer. Clin Endocrinol. 2003; 58:400-402.

35. Gharib H, Papini E, Paschke R, Duick DS, Valcavi R, Hegedüs L, Vitti P. American Association of Clinical Endocrinologists, Associazione Medici Endocrinologi, and European Thyroid Association Medical Guidelines for Clinical Practice for the Diagnosis and Management of Thyroid Nodules: Executive Summary of Recommendations. J Endocrinol Invest. 2010; 33:287-291.

36. Wells BS GA, O’Connell D, Peterson J, Welch V, Losos M, Tugwell P. The Newcastle-Ottawa Scale (NOS) for assessing the quality of non-randomized studies in metaanalyses. 2013. 
37. Higgins JP, Thompson SG. Quantifying heterogeneity in a meta-analysis. Stat Med. 2002; 21:1539-1558.

38. DerSimonian R, Laird N. Meta-analysis in clinical trials. Control Clin Trials. 1986; 7:177-188.

39. Greenland S, Longnecker MP. Methods for trend estimation from summarized dose-response data, with applications to meta-analysis. Am J Epidemiol. 1992; 135:1301-1309.
40. Egger M, Davey Smith G, Schneider M, Minder C. Bias in meta-analysis detected by a simple, graphical test. BMJ. 1997; 315:629-634. 